\title{
Diversidad cultural y fomento de valores en la convivencia ciudadana
}

\author{
Cultural diversity to promote values in the life of society
}

Sonia Ethel-Durán ${ }^{1}$, Jesús Enrique García-Guiliany², Margel Parra-Fernández ${ }^{3}$

\begin{abstract}
${ }^{1}$ Doctora en ciencias gerenciales, socióloga. Investigadora senior. Docente investigadora. Identificador de autora: http://orcid.org/ 0000-0002-0876-7850; correo: soniaduran547@hotmail.com

${ }^{2}$ Doctor en ciencias gerenciales, investigador senior y docente investigador, Universidad Simón Bolívar, Barranquilla (Colombia). Identificador de autor: http://orcid.org/ 0000-0003-3777-3667; correo: jesus. garcia@unisimonbolivar.edu.co

${ }^{3}$ Doctoranda en formación, magíster en gerencia de recursos humanos, psicóloga, técnica superior universitaria en educación preescolar. Investigadora asociada. Docente e investigadora vinculada al Grupo de Investigación en Pedagogía, Corporación Universitaria Latinoamericana (CUL), Barranquilla (Colombia). Identificador de autora: https://www.researchgate.net/profile/Margel_Parra; correo: margel. alejandra@gmail.com
\end{abstract}

Recibido: 11-10-2017; Aceptado: 01-11-2017

\section{Resumen}

La investigación se orientó a evaluar la diversidad cultural para el fomento de valores en la convivencia ciudadana, la metodología fue descriptiva, con un diseño no experimental de campo. La población fue de doscientas cincuenta personas. Se aplicó un cuestionario constituido con 37 ítems con alternativas tipo escala. Los principales resultados indican que para el fomento de los valores en la convivencia ciudadana es necesario gestionar la diversidad cultural, lo cual permitirá canalizar las diferencias socioculturales y religiosas que pudieran presentarse durante el proceso de convivencia. Dado que las personas víctima del conflicto armado pueden asumir procesos hostiles y dificultad para la integración, se hacen relevantes las diferencias entre ellos, y es necesaria la promoción de valores para fomentar una sociedad basada en principios éticos, de manera que se eviten las conductas negativas, que perjudiquen el bienestar interior del individuo.

Palabras clave: diversidad cultural, valores, convivencia ciudadana. 
Ethel-Durán et al. Diversidad cultural y fomento de valores en la convivencia ciudadana

\section{Abstract}

The research was aimed at evaluating the cultural diversity for the promotion of values in the life of society, the methodology was descriptive, with a non-experimental design field, the population was 250 people. A questionnaire consisting of 37 items with alternative type scale was applied. The main results indicate that for the promotion of the values in the life of society is necessary to manage cultural diversity, to channel differences, socio-cultural and religious that might arise during the process of coexistence. Since people victims of the armed conflict can take hostile processes and difficulty in integration, differences between them are relevant, and it is necessary to promote values for a society based on ethical principles, avoiding negative behaviors that adversely affect the domestic welfare of the individual.

Key words: cultural diversity, values, citizen coexistence.

\section{Introducción}

En la actualidad, las sociedades se encuentran inmersas en un proceso de cambio continuo, en ocasiones incoherente, situación producida por una cantidad de conflictos que se han generado desde las comunidades $y$ hacia ellas. Dentro de este marco, la compleja y crítica realidad en materia de convivencia, producto de violencia, violaciones de los Derechos Humanos, guerra, paz inconclusa y conflictos, obliga a los actores sociales a intervenir de alguna forma y generar un aporte para desencadenar procesos complejos positivos hacia las comunidades víctimas de este conflicto. Debido a la poca o nula capacidad de regulación estatal de los conflictos, surge la necesidad de plantear procesos en los que la sociedad participe y haga aportes de tipo institucional y social, con miras a mejorar la calidad de vida de dichas comunidades.

En Colombia existen diversos factores de riesgo a los que está expuesta mayoritariamente la población afectada por el conflicto armado, pues está inmersa en una serie de eventos significativos que potencialmente pueden poner en riesgo la integridad física y psicológica del individuo. Estos pueden ser resultado tanto de violencia directa como cultural o estructural. No obstante, en los últimos años se ha percibido, a pesar de los contrastes en el escenario estratégico y los retos para superar el conflicto y consolidar la paz, que los problemas y conflictos siguen siendo enormes. Ciertamente la sociedad se ha aliviado por la mejora radical de las condiciones de seguridad, pero el fenómeno del narcotráfico, que por años ha ali-mentado a los actores armados irregulares, no cede tanto como se esperaría, dado el descomunal esfuerzo empeñado en ello.

En este sentido, cuestiones como las tensiones en el escenario latinoamericano; su incidencia en el conflicto armado colombiano y las alternativas de política exterior para Colombia, y los avances del proceso de desarme, desmovilización y reintegración de los grupos paramilitares y sus reveses especialmente en lo relativo al rearme de reductos de estos grupos se mantienen hasta el presente en el centro del debate de la guerra y la paz en Colombia. Bajo este contexto, en el ejercicio de la convivencia ciudadana, y en la búsqueda de marcar la diferencia en la manera de crear cultura social y comunitaria, Duran, Fuenmayor, Cárdenas y Hernández (2016) han manifestado que para afianzar el sentido de pertenencia 
e integración comunal, las instituciones del Estado encaminadas al trabajo con las comunidades en condiciones de vulnerabilidad se han orientado a fomentar valores sociales e individuales para generar un proceso de integración, como un sistema de direccionamiento de políticas de desarrollo social y personal. Esto para promover el desarrollo de las comunidades, sus procesos personales y sociales, a partir de la base de la diversidad cultural.

Esta situación debe concretarse, según Montesinos (2016) para que estos se adapten a los cambios culturales y sociales originados por el proceso de globalización. Cabe destacar que las organizaciones e instituciones públicas y privadas, según Prieto y Hernández (2016) han asumido el rol de responder a las necesidades sociales, preocupándose por estandarizar procesos de convivencia, de integración e inserción social. Dentro de este marco, se vislumbra la necesidad de responder a los lineamientos de cooperación, calidad y preservación del entorno.

En este sentido, la investigación tiene como finalidad establecer herramientas y desarrollar tácticas para fomentar valores para la convivencia ciudadana, tomando como inicio la diversidad cultural de los miembros de las comunidades, con el objetivo de promover la interacción e integración positiva entre todos sus miembros, facilitando el progreso y avance de las comunidades integradas, dada la importancia que representa la gestión social de las instituciones que tienen esa labor en las comunidades. Para ello requiere desarrollar una profunda conciencia histórica que permita comprender la trascendencia generacional de los actos humanos.

Además de orientar al ciudadano para respetar el medio ambiente social y cultural en función de los valores, implica entender que lo que hace una generación se lo hereda a la que sigue. Por otra parte fomentar el consumo inteligente, moderado y crítico, tanto de los bienes y servicios como de la información que manejan con ética y honestidad.

La idea principal es que los ciudadanos sean capaces de utilizar creativa y productivamente su tiempo libre. Promoviendo el desarrollo social, deportivo y cultural de sus espacios de vida, de tal manera que se fomente el servicio a la comunidad, para el servicio a los demás. Conocedores y respetuosos de los Derechos Humanos, se busca que valoren la vida y la paz, y formarlos en la resolución no violenta de conflictos. La idea es buscar la paz y la vigencia de un estado de derecho respetuoso de los Derechos Humanos.

Esto debido a que en el diario vivir se ven abocados a manejar e intentar resolver, bajo algún criterio de justicia, graves conflictos y múltiples manifestaciones de violencia que de una u otra manera alteran la convivencia ciudadana o rompen, en muchas comunidades del país, sus posibilidades de crecimiento $y$ desarrollo. En virtud de que el conflicto es una situación que se presenta en estas sociedades de una forma brusca y que ha truncado las oportunidades, se debe buscar armonizar actitudes, intereses y propósitos diferentes con algunas alternativas viables.

En opinión de Duran, Prieto y García (2017) entre las condiciones de vida de las personas que propenden por la calidad de la misma, se pueden resaltar la disponibilidad de fuentes de trabajo, condiciones de vivienda, servicios de atención médica, disponibilidad de alimentos, existencia de centros culturales, deportivos, saneamiento ambiental, transporte y comunicaciones, entre otros. Se destaca, no obstante, que 
las comunidades en estudios, están muy lejos de ese proceso de calidad de vida, pues tienen un proceso generado como consecuencia del conflicto armado, las cuales además han sido afectados social, económica y ambientalmente por todos esos procedimientos que han ocasionado un detrimento en su vida diaria y en sus familias.

Se han buscado entonces alternativas que ayuden a estas poblaciones a superar de alguna manera las limitaciones y problemas consecuencia de dichos conflictos. Lo que se pretende es generar y propiciar la integración de los ciudadanos desde el fomento de valores, siendo necesario comprenderla desde diferentes perspectivas y realidades (García, Duran, Parra y Márceles, 2017). En este sentido, el estudio sugiere gestionar la diversidad cultural, dado que en la actualidad, se habla de interculturalidad de sociedades multicolores, que según Duran (2014) don aquellas donde conviven personas con diversidad racial y cultural, en las cuales se congregan diversos credos, ideologías, así como las diferentes costumbres.

Dentrodeestemarco, Duran y Parra(2014) consideran que, para la supervivencia y prosperidad en una sociedad heterogénea cada vez más compleja, se debe manejar la diversidad entre los ciudadanos con opciones positivas, de lo contrario pueden crearse malos entendidos que tendrán un impacto negativo en la integración y socialización. Existe la necesidad de desarrollar estrategias para promover con efectividad y calidad en el ambiente donde se desenvuelven, para lo cual el ciudadano representa uno de los factores más importante, sumado esto a los cambios estratégicos que deben estar apoyados por los valores, actitudes y conductas de su gente; ello permite fomentar la convivencia ciudadana, en pro de alcanzar los objetivos de las comunidades.
Cabe destacar que tomar en cuenta la diversidad cultural según Duran (2010) puede incidir positivamente en la disminución de los conflictos, mejorar el proceso social, estimulando la creatividad, mejorar la resolución de problemas, y dar más flexibilidad. Por lo tanto, se debe pensar en la adaptación, disposición o preparación de los ciudadanos para beneficiarse de ellos, ajustándolos a la especificidad de cada situación, organización y grupo cultural.

En este orden de ideas, Robbins (2014) sostiene que la diversidad cultural se relaciona con la heterogeneidad existente en término de raza, cultura, religión, sexo, origen étnico y discapacidad. Además, producto de la globalización, el mundo dista de ser homogéneo, por ende, el camino se ha ampliado y se debe promover la equidad y el respeto, así como la justicia social. Relacionado con los valores, García et al. (2017) sustentan que este aspecto representa un factor determinante para la constante dinámica de desarrollo y evolución de la sociedad, incluida en todos sus niveles. Dado que los cambios en el comportamiento del individuo han venido paralelos a un cambio en el perfil y vida sociocultural y laboral.

Esto implica realizar un conjunto de estrategias y procesos para lograr dirigir su aplicación, ayudando en la convivencia ciudadana, al logro de sus objetivos y metas propuestas. En tal sentido, la comprensión de la práctica social, así como los valores que la sustentan, en espacios sociales y comunitarios específicos, emergen desde el seno de la familia, se sustentan en el trabajo, manteniéndose en el transcurso de las décadas, representativos de una parte del colectivo, como parte esencial de la identidad social, asumida por el individuo.

Se hace necesario que el ciudadano asuma una conducta fundamentada en valores 
personales para proveer su acción con valores sociales. Considerando la apertura del nuevo milenio, la cual abre un compás hacia un nuevo esquema y diseño de desarrollo social, los valores se manifiestan en las acciones de las personas; en sus palabras. Por tanto, a través de las acciones repetitivas y constantes que manifiestan un colectivo de personas, la investigación parte de una necesidad de un grupo como son las personas que viven en condiciones de vulnerabilidad.

De acuerdo a lo planteado, surgió la necesidad de evaluar la gestión de la diversidad cultural para el fomento de valores en la convivencia ciudadana.

\section{Perspectiva de la diversidad cultural}

La diversidad cultural es un sistema de principios y de juicios compartidos por los conceptos, las creencias culturales, religiosas y filosóficas, lo cual determina si algunas acciones dadas son correctas o incorrectas. Estos conceptos son generalizados, además suelen se codificados a menudo por una cultura o un grupo y sirven así para regular el comportamiento de sus miembros independiente de sus consecuencias. Se destaca que la diversidad abarca sexo, edad, lenguaje, etnia, antecedente cultural, orientación sexual, creencia religiosa y responsabilidad familiar; también se refiere en otro modo a la forma en la cual la gente es diferente, como el nivel educacional, experiencias de vida y de trabajo, antecedente socioeconómico, personalidad y estado civil. Involucra el reconocimiento del valor individual de las diferencias.

De acuerdo a lo planteado, Newstrom (2011) considera que la diversidad cultural se manifiesta por la diversidad del lenguaje, las creencias religiosas, las prácticas del manejo de los valores, en el arte, en la música, en la estructura social, en la dieta y en todo número concebible de otros atributos de la sociedad humana. Dado que el individuo vive en un mundo de continuos cambios sociales, tecnológicos, económicos, políticos y culturales, los elementos que rodean e influyen al ser humano evolucionan; de igual manera él lo hace también.

Por otra parte, las personas buscan mejores oportunidades de estudio, de trabajo, con la motivación de conseguir mejor situación económica, social y una buena calidad de vida. A este respecto, Newstron (2011) afirma que las personas emigran de sus países de origen, se mudan de ciudad, trabajan en varios empleos, sacrifican muchas cosas para hacer realidad sus sueños. Los movimientos migratorios han retado a las sociedades y a las organizaciones empresariales y gubernamentales a tratar con personas de diferentes nacionalidades y grupos étnicos, culturales y religiosos, a tener que legislar sus países de una forma diferente, sobre todo, a tenerlos que integrar en la sociedad para que sean productivos.

Kreitner y Kinicki (2006) sostienen que el fenómeno de la globalización implica, flujos migratorios e intercambios culturales que sitúan al individuo en un claro contexto de interculturalidad. Pero este proceso de aumento de la diversidad social no se refiere exclusivamente a diferencias culturales, sino que hace referencia también a otros grupos sociales hasta ahora considerados minoritarios.

Este cambio social tiene sus repercusiones en todos los aspectos de la vida, situación que afecta los procesos económicos y gubernamentales: la edad media de los empleados aumenta, crece el acceso de las mujeres al mercado de trabajo, de la misma forma, se hacen más evidentes los distintos grupos étnicos, culturales o religiosos que conviven en las sociedades, personas con diversidad funcional o alguna discapacidad y grupos con diferente orientación sexual, de los 
que se originan los grupos LGBT. Ahora bien, ¿cómo destacar el proceso de convivencia de diferentes grupos según los valores que hayan adquirido durante su crecimiento y desarrollo psico-social?.

\section{Formación en valores}

Todaslaspersonastienen unos valoresque les guían consciente o inconscientemente. El proyecto de vida individual y colectiva se diseña a través de estos valores, que están ligados a aspectos culturales y vivencias personales, aunque las diversas trayectorias individuales, sociales o profesionales, la diferencia de informaciones percibidas por cada uno, entre otros aspectos, conducen a no tener la misma percepción del mundo. De acuerdo con ello, Martinneli (2004) sostiene que los valores son el conjunto de reglas de conducta, de leyes juzgadas conforme a un ideal, para una persona o colectividad.

En este contexto, los valores representan una forma de vivir. Ellos están relacionados con la identidad de la persona, con el medio y con la reflexión. Un valor es una toma de posición relacionada a cualquier cosa, experimentada a través de comportamientos, sentimientos, conocimientos, y acciones. Es así como el concepto de valor, en opinión de Cortina (2007), puede definirse como una manera de ser o de obrar, que una persona o una colectividad juzgan ideal, y que hace deseables o estimables a los seres o a las conductas a las que se atribuye dicho valor. El concepto valor va unido a una serie de características; el valor está en el orden ideal, los objetos y acontecimientos sirven de inspiración y referencia a un determinado orden moral, estético o intelectual. El valor denota cualidad de ser, a la que se aspira y en la que cabe inspirarse; el valor está inmerso en la sociedad, formando parte de ella.
Por otra parte, Cortina (2007) expresa que los valores son necesarios para hacer habitable el mundo. En esa medida, los considera "imprescindibles para una sociedad plural y democrática, libertad, igualdad, solidaridad, responsabilidad, respeto activo y disponibilidad al diálogo". Según la autora, estos valores pertenecen a la plenitud de lo humano, son libres; depende de cada persona, el encarnarlos o no, pero también son exigibles. Señala además que la educación en valores es una necesidad para construir, consolidar y mantener una sociedad pluralista y democrática, porque educar en valores es bueno, positivo, no se debe caer en complejos heredados de regímenes anteriores; si no se educa en estos valores, no se educa humanamente.

Loanteriormenteexpuestopermite deducir que en la educación se debe enseñar en valores, para contribuir a fomentar una sociedad basada en principios éticos y evitar caer en conductas negativas, que perjudiquen el bienestar interior del individuo. En cuanto a los valores, se requiere un equilibrio, es decir que cada valor tomado individualmente sea saludable; el valor nace y se desarrolla desde la familia. Por ende, es necesario descubrir la raíz que hace de la familia el lugar ideal para forjar esos valores, para así darle continuidad a lo largo de la vida del individuo en todas las etapas, en su formación académica, desempeño profesional y personal, en que la ética es una meta alcanzable y necesaria para lograr un modo de vida más humano, que posteriormente se transmitirá naturalmente a la sociedad entera.

Dentro de este marco, los valores se van adquiriendo y moldean a lo largo de la vida, no se enseñan con palabras, son transmitidos por contacto, siendo posible convertirlos en vivencia personal, cuando se desea y aprecia para la propia vida. También influyen en su internalización las 
actitudes y maneras de pensar de otras personas, pues estas despiertan el interés para hacer que estos puedan desarrollar el deseo de realización personal, es decir, observando modelos a imitar para cimentar una mejor satisfacción de vida.

En consecuencia, los valores contribuyen en gran medida a la orientación de una vida en la cual el individuo se pueda desarrollar plenamente como persona. Aunque son complejos, todos los valores coinciden en que tienen como fin último mejorar la calidad de vida. Dentro de este marco, Alonso (2004) esboza que un sistema de valores es una organización de valores, en la cual cada uno de estos se alinea a lo largo de un proceso continuo, generando, según su importancia, una escala de valores, esto es, la jerarquía de los mismos. Bajo este contexto, los valores están ordenados jerárquicamente, según se trate de una u otra sociedad; por consiguiente ellos pueden combinarse e interactuar.

\section{Convivencia ciudadana}

Una de las estrategias que puede implementar el promotor de la formación de valores es la convivencia, entendida por Rodríguez (2004) como la interrelación entre los diferentes miembros de un establecimiento educacional, que tiene incidencia significativa en su desarrollo ético, socio afectivo e intelectual. Esta concepción no se limita a la relación entre las personas, sino que incluye las formas de interacción entre los diferentes estamentos que conforman una comunidad, por lo que constituye una construcción colectiva y es responsabilidad de todos los miembros y actores sociales sin excepción.

En todo caso, se constituye un aporte decisivo que contribuye a la calidad en la formación en valores, constituyéndose un espacio privilegiado de intercambio personal, social y organizacional, que sirve de modelo a los estilos de relación entre sus miembros. Por ello, una de las formas de avanzar en la construcción de una sociedad sana y sustentable, como lo han expresado Duran, Parra y Márceles (2015), es reconocer la importancia de la acción del docente en el ámbito de la convivencia, específicamente relacionada con la formación en valores, lo cual influye en el desarrollo de habilidades sociales. Desde esta perspectiva, se espera que el ciudadano mismo sea capaz de crear instancias de reflexión y consulta en todos los actores sociales y educativos, sobre aspectos de la convivencia ciudadana que inciden directamente en el proceso de la enseñanza de valores.

En consecuencia, los valores sociales son los pilares que sustentan el comportamiento y las formas de vida de los seres humanos. Constituyen un elemento decisivo en la vida de los grupos humanos. Martinneli (2004) afirma que con los valores se involucra la dignidad de la persona, en tanto que esta se desarrolla, crece, se realiza individual y colectivamente, respeto a sus valores a sus diferencias, confidencialidad, respeto a sus derechos y libertades. Se trata del reconocimiento del potencial humano de la persona: de cambio, de competencia, de capacidad de adaptación, de capacidad de interrelación con otros y con el entorno, capacidad de auto cuidarse y potencial de auto curación.

En este sentido, la persona merece respeto a su derecho a la autodeterminación: es decir, auto responsabilidad, toma de decisiones, participación. La persona es vista como un sistema abierto y parte integrante de sistemas más grandes (familia, sociedad, entorno, etc.). También como un ser global e indisociable; como un ser entero biopsicosocial-cultural tal como lo han expresado Duran (2010) y Duran y Parra (2014): capaz de percibir, de comprender, de interpretar las 
situaciones y libre de actuar. Dentro de este marco, un entorno sano, constituido por un conjunto de elementos del medio, contribuye al desarrollo de las personas, puesto que las condiciones relativas al contexto prevalecen e interactúan con ellas, influyendo en su salud y en su bienestar. De igual manera la interdependencia, la permeabilidad de la materia y de la energía entre la persona y el medio.

En tal sentido, los valores son guías determinantes de actitudes sociales e ideologías, en las que las actitudes son tendencias a comportarse de una forma consistente y persistente delante de una determinada situación, objeto, hecho o persona. Las actitudes traducen, a nivel de conducta, el mayor o menor respeto o adherencia a unos determinados valores.

\section{Metodología}

El objetivo de la investigación fue evaluar la gestión de la diversidad cultural para el fomento de valores en la convivencia ciudadana. Esta investigación es de tipo descriptiva, ya que describe los elementos que la conforman. En cuanto al diseño concebido como la estrategia que adopta el investigador, fue no experimental, ya que se gestionó la diversidad cultural para fomentar los valores sin participar o manejar el contexto, y se ejecutó una sola medición. Asimismo, es de campo, ya que la información y los datos fueron obtenidos directamente en las comunidades seleccionadas.

Respecto a la población, esta se constituye por características o estratos que le permiten distinguir los sujetos unos de otros, ellas deben ser delimitadas con el fin de establecer los parámetros muéstrales y se incluyen en estas la totalidad de los sujetos, objetos, fenómenos o situaciones a investigar. Este estudio se sustentó con la propuesta de Parra (2006), quien plantea que la muestra puede ser probabilística y no probabilística (como el caso de la intencional). Se concibe el muestreo intencional o por conveniencia, la cual se determina como la selección de los elementos que se deja a juicio de los investigadores y a quienes aplican el cuestionario. Estos seleccionan los elementos dada la facilidad de acceso o conveniencia.

En este sentido, la investigación tuvo como base muestral intencional doscientas cincuenta personas (habitantes de la comunidad de Villa Gardenia en el distrito Barranquilla), quienes fueron distribuidos de forma estratificada según la cantidad total de personas que viven en la comunidad seleccionada. Dichas personas asistieron a las plazas y lugares públicos, los días de la aplicación, las cuales fueron abordadas en días de mayor movimiento, hasta completar la cifra establecida. Estos días fueron los fines de semanas.

Tabla 1. Muestra intencional

\begin{tabular}{|c|c|c|}
\hline $\begin{array}{c}\text { Comunidad } \\
\text { Villa Gardenia }\end{array}$ & \% & $\begin{array}{c}\text { Muestra } \\
\text { intencional }\end{array}$ \\
\hline Semana 1 & $65,4 \%$ & 164 \\
\hline Semana 2 & $34,6 \%$ & 86 \\
\hline Total & $100 \%$ & 250 \\
\hline
\end{tabular}

Fuente: Elaboración propia (2017)

Consecuentemente, se hizo el proceso de recolección de datos, el cual se realizó a través de la técnica de encuesta y la aplicación de un cuestionario, que estuvo conformado por 37 ítems de escala Likert, en función de los elementos y variable. 
Tabla 2. Escala de respuesta

\begin{tabular}{|c|c|}
\hline RESPUESTA & VALOR \\
\hline Totalmente de acuerdo & 5 \\
\hline De acuerdo & 4 \\
\hline Más o menos de acuerdo & 3 \\
\hline Desacuerdo & 2 \\
\hline Totalmente en desacuerdo & 1 \\
\hline
\end{tabular}

Fuente: Elaboración propia (2017)

Posteriormente, se realizó el análisis de los datos, que consistió en registrar sistemáticamente las respuestas emitidas porel grupo muestral. Se les codifico con un número para darle sustento al tratamiento estadístico. A efectos de la presente investigación, se asumió la estadística inferencial como el procedimiento que facilita el análisis e interpretación de los resultados obtenidos a través de la fase de recolección. Los elementos planteados facilitaron la presentación de los resultados de investigación.

\section{Resultados}

El análisis de los resultados se realizó a través de la estadística descriptiva, los datos han sido representados en forma de frecuencia (FA) y porcentajes (\%), es decir; en un conjunto de puntuaciones ordenadas en sus respectivas categorías, complementadas con las frecuencias relativas, las cuales representan los porcentajes de casos en cada categoría y con evaluación del promedio.

Tabla 3. Proceso de convivencia

\begin{tabular}{|c|c|c|c|c|c|}
\hline \multicolumn{6}{|c|}{ Respuesta escala Likert } \\
\hline Indicadores & $\begin{array}{l}\text { Totalmente de } \\
\text { acuerdo }\end{array}$ & De acuerdo & $\begin{array}{c}\text { Más o menos de } \\
\text { acuerdo }\end{array}$ & Desacuerdo & $\begin{array}{c}\text { Totalmente en } \\
\text { desacuerdo }\end{array}$ \\
\hline Cooperación & 20,4 & 49,8 & 27 & 2,8 & 0 \\
\hline Respeto & 40,2 & 13 & 10,4 & 22,8 & 13,6 \\
\hline Justicia & 18,4 & 17,2 & 25,6 & 32 & 6,8 \\
\hline Paz & 27,8 & 40,4 & 10 & 17 & 4,8 \\
\hline $\begin{array}{l}\text { PROMEDIO DI- } \\
\text { MENSIÓN }\end{array}$ & 26,7 & 30,1 & 18,25 & 18,65 & 6,3 \\
\hline
\end{tabular}

5: Totalmente de acuerdo. 4: De Acuerdo: 3. Más o menos de acuerdo. 2: Desacuerdo. 1: Totalmente en Desacuerdo. Fuente: Elaboración propia (2017)

Con respecto a la situación actual del proceso de convivencia, se puede observar, en la cooperación, que el $49 \%$ y el $20,4 \%$ se inclinan hacia las existencias de cierta cooperación, sin embargo, un $29 \%$ de la población encuestada considera estar en desacuerdo con que estas comunidades tienen cooperación en su convivencia. Por otra parte, se analizó el respeto. En este ámbito se demuestra que el $53 \%$ de la población estuvo entre totalmente de acuerdo y de acuerdo con el hecho de que estas comunidades presentan debilidades en el respeto a la convivencia ciudadana. No obstante, un número bastante importante (35\%) sostuvo que las situaciones planteadas u ocurridas no están referidas a que no exista el respeto.

En el mismo orden de ideas, la mayoría de la población consideró que las situaciones planteadas representan un bajo nivel de justicia, en virtud de representar el 35\% de la población encuestada, además de que un $25,6 \%$ manifestó estar más o menos de acuerdo, lo cual se inclina hacia la posibilidad de seguir en la búsqueda de 
Ethel-Durán et al. Diversidad cultural y fomento de valores en la convivencia ciudadana

la justicia social, moral y legal, generando la posibilidad de fomentar la convivencia y lograr los objetivos de las comunidades organizadas. Seguidamente se presenta la paz, en cuyo ámbito hay una proporción muy alta que plantea la necesidad de que haya paz (casi el $60 \%$ de la población), además, un $18,25 \%$ sostuvo estar más o menos de acuerdo.
En este panorama, se estima que la situación actual de las comunidades en el proceso de convivencia está condicionada por el movimiento migratorio, por las movilizaciones y la alta situación de carencias que existe en la actualidad, sin embargo, se presenta una posibilidad de querer participar en procesos que forjen los valores, tomando en cuenta la diversidad cultural y social.

Tabla 4. Problemas de las comunidades

\begin{tabular}{cccccc}
\hline Indicadores & $\begin{array}{c}\text { Totalmente de } \\
\text { acuerdo }\end{array}$ & De acuerdo & $\begin{array}{c}\text { Más o menos de } \\
\text { acuerdo }\end{array}$ & $\begin{array}{c}\text { Desacuerdo } \\
\text { Totalmente en } \\
\text { desacuerdo }\end{array}$ \\
\hline Desconfianza & 5,6 & 33,6 & 38,6 & 13,8 & 8,4 \\
Seguridad & 0,6 & 40,4 & 25 & 32 & 2 \\
Sensibilidades & 21,8 & 59,2 & 17,2 & 1,6 & 0,2 \\
Integración comunitaria & 14,8 & 33,6 & 21,2 & 30,4 & 0 \\
Empatía & 23,4 & 21,8 & 17,4 & 30,8 & 6,6 \\
\hline PROMEDIO & 13,24 & 37,72 & 23,88 & 21,72 & 3,44 \\
DIMENSIÓN & & &
\end{tabular}

5: Totalmente de acuerdo. 4: De acuerdo. 3: Más o menos de acuerdo. 2: Desacuerdo. 1: Totalmente en desacuerdo. Fuente: Elaboración propia (2017)

La tabla 4 muestra la información sobre los elementos que se tomaron en cuenta en esta dimensión, en los que se ha considerado, según los individuos encuestados, que en las comunidades se vive con alta desconfianza. Esto está representado por el $38,6 \%$ en la posición más o menos de acuerdo, un $33,6 \%$ de acuerdo y un 5,6\% totalmente de acuerdo. No obstante, un grupo importante del $13,8 \%$ estuvo en desacuerdo y un $8,4 \%$ en total desacuerdo.

Seguidamente, en cuanto a la seguridad, se consideró la existencia de esta en el quehacer diario y se planteó si los vecinos - personas alrededor les apoyarían cuando ocurriese una situación irregular: el $40,4 \%$ estuvo de acuerdo con ello y un $25 \%$ más o menos de acuerdo; por otro lado, un $32 \%$ estuvo en desacuerdo. Así pues, las tendencias se inclinan hacia la falta de seguridad.

Con respecto a las sensibilidades, la mayoría considera que el grueso de las personas se orienta a respetar las sensibilidades de los vecinos: $21,8 \%$ estuvo totalmente de acuerdo, un $59,2 \%$ estuvo de acuerdo. Seguidamente se analiza la integración comunitaria, aspecto en el que se obtuvo que un $33,6 \%$ estuvo de acuerdo con este aspecto; no obstante, un $30,4 \%$ estuvo en desacuerdo, un $21,2 \%$ consideró estar más o menos de acuerdo y un $14,8 \%$ estuvo totalmente de acuerdo. Las tendencias se inclinan hacia una integración comunitaria efectiva o esperada por los miembros de la comunidad. 
En el ámbito de la empatía, la tendencia es variables, ya que el mayor porcentaje de $30,8 \%$ consideró estar en desacuerdo, un $23,4 \%$ y un $21,8 \%$ manifestaron estar totalmente de acuerdo y de acuerdo, respectivamente. Por otra parte, un $17,4 \%$ estuvo más o menos de acuerdo.
En este caso las tendencias indican la existencia de cierta empatía hacia el vecino, dado que pocas veces existe igualdad entre los miembros, además de que el respeto activo debe fomentarse desde las bases de la familia.

Tabla 5. Valores que manifiestan el comportamiento de los integrantes de la comunidad

\begin{tabular}{|c|c|c|c|c|c|c|c|}
\hline Dimensión & \multicolumn{6}{|c|}{ Valores que manifiestan el comportamiento de los integrantes de la comunidad } \\
\hline Indicador & Cooperación & Respeto & Justicia & Paz & Libertad & Responsabilidad & Honestidad \\
\hline Promedio & 3,5 & 2,9 & 2.4 & 4,6 & 4,7 & 4,5 & 3,8 \\
\hline Moda & 4 & 3 & 2 & 5 & 5 & 5 & 5 \\
\hline Mediana & 4 & 3 & 2 & 5 & 5 & 5 & 5 \\
\hline $\begin{array}{c}\text { Desviación } \\
\text { estándar }\end{array}$ & 1,2 & 1,1 & 1,2 & 0,9 & 0,8 & 1,0 & 1,6 \\
\hline
\end{tabular}

Fuente: Elaboración propia (2017)

Siguiendo con el análisis, la tabla 5 presenta las estadísticas descriptivas para los indicadores de la dimensión y valores que manifiestan el comportamiento de los integrantes de la comunidad. Se encontró con respecto al indicador de cooperación una media aritmética de 3,5 , de manera que se asume, según el baremo, una cooperación eficiente. El valor representado en la moda fue el 5 , la mediana se ubicó en 4 y la desviación estándar en 1,2 , lo que demuestra entonces una dispersión muy baja de los puntajes con respecto al promedio.

Para el indicador de respeto, el promedio se ubicó en 2,9 , y se observó como un valor poco eficiente. Asimismo, la moda y la mediana fueron de 3 y la desviación estándar fue de 1.1, lo que implica una dispersión baja de los puntajes con respecto al promedio. Con respecto al indicador justicia, el promedio fue de 2, 4, que se interpreta como poco eficiente; la moda y la mediana fueron de 2 , mostrando una tendencia hacia valores negativos. Por otro lado, la desviación estándar fue de 1,2 , lo que indica una dispersión baja de los puntajes en relación al promedio.

Para el indicador paz, el promedio se ubicó en 4,6, lo que se interpreta como un valor muy eficiente. Mientras la moda y la mediana se ubicaron en 5 , la desviación estándar fue de 0,9 , lo que implica una dispersión muy baja de los datos en relación al promedio. Con relación al indicador libertad, el promedio fue de 4,7 , lo que se considera muy eficiente; la moda fue de 5 , la mediana fue de 5 y la desviación estándar fue de 0,8, lo que significa que hay una dispersión muy baja en relación al promedio.

Asimismo, para el indicador responsabilidad, el promedio se ubicó en 4,5 , muy eficiente; la moda y la mediana fueron de 5, y la desviación estándar fue de 1 , lo que representa una muy baja dispersión de los datos con relación al promedio. Al analizar el indicador honestidad, se observó un promedio de 3,8 , que se interpreta como un valor eficiente, la moda fue de 5 , la mediana 
asumió el mismo valor, mientras la desviación estándar fue de 1,6 , lo que indica una dispersión baja de los puntajes en correspondencia con el promedio.

Los valores se transmiten por contacto, la interacción de las personas en las comunidades y en todos los estamentos de la sociedad, en su convivir, son muy importantes, además influyen en su internalización las actitudes y maneras de pensar de los involucrados en este aspecto, lo que despierta el interés para hacer que estos puedan desarrollar el deseo de realización personal, es decir, que se observan modelos a imitar para cimentar una mejor satisfacción de vida.

Para Esté (2002), los valores constituyen herramientas que los seres humanos utilizan para perfilar sus creaciones y sus maneras de comprender subjetivamente. En este sentido, el individuo y el grupo construyen sus aprendizajes, conocimientos y proyectos. Pueden tener un papel positivo o negativo, pero siempre de gran vigencia; positivo cuando el conocimiento puede estar referido convergentemente en ellos y negativo, si el conocimiento puede referirse a ellos, pero en todo caso, el valor firmemente establecido es necesario para lo que se acepte como verdadero o deseable.

Por lo tanto, los ciudadanos y los involucrados en la formación diaria pueden ayudar a que los individuos desarrollen los valores. En el trabajo educativo formal e informal, la imaginación creadora juega un papel importante a este respecto. Así, el promotor debe ser capaz de presentar el atractivo del bien, de la honestidad, de la comunicación, de la sencillez de la vida, del compartir, del perdonar; en suma, de todos los valores que han pasado a un segundo plano o prácticamente han desaparecido, por lo que la educación debe ser capaz de generar y de presentar modelos inspiradores.
En cuanto al indicador cooperación, se manifiesta en actividades cotidianas que están repletas de valores, ello indica aspectos positivos hacia las comunidades y sus miembros, en este sentido, Carreras (2000) asume la cooperación como la acción que se realiza conjuntamente con otro y otros individuos para conseguir un mismo fin. Esta acción, aunque a veces es un beneficio para sí mismo, siempre tiende a beneficiar a los demás. Se infiere, por tanto, que para que este acto se considere cooperativo tiene que existir una reciprocidad, si no existe esta, no se puede hablar de cooperación, sino solo de ayuda.

Para el indicador respeto, el promedio se ubicó en 2,9 , es decir que este valor no se promueve, desde la práctica social y cultural, por ende, deben observarse las líneas que se están aplicando a este respecto, pues este es un valor muy importante en el desarrollo y promoción de valores sociales. Bajo este contexto, Esté (2002) considera que el respeto "significa responsabilidad hacia la persona misma, esto a la vez significa ser libre". De acuerdo con el autor, en el quehacer sociocultural los diferentes estamentos deben poseer conciencia moral de sí mismos, porque la personalidad moral es auténtica. Sin conciencia moral el respeto carece de dignidad, de amor hacia sí mismo y los demás.

Con respecto a la justicia, el promedio fue de 2,4, que se interpreta como poco eficiente, es decir que tiene una tendencia baja con respecto a su promoción en la práctica social. En este ámbito se cita a Carreras (2000), quien asume que es la actitud moral o voluntad decidida de dar a cada uno lo que es suyo, por lo tanto, es un valor que supone siempre, a la otra persona a quien debe respetarse.

Además, es la virtud de la equidad, la igualdad y el orden. De allí que conocer 
este valor sea fundamental, porque es la base de otros valores. A este respecto, deben ejecutarse actividades como educar a los niños y jóvenes en la práctica y conocimiento de la justicia, tanto en la clase como en su relación con los demás y enseñar a aceptar y respetar las normas siendo equitativo.

Por otro lado, se percibe el indicador paz, en el que el promedio se ubicó en 4,6 , lo que se interpreta como muy eficiente, es decir que se promueve la paz en las comunidades a pesar de no promoverse la justicia. De acuerdo con esto se asume la posición de Carreras (2000), quien manifiesta que la persona que goza de paz se caracteriza por poseer un equilibrio psíquico, sentimientos altruistas y ausencia de conflictos entre su corazón y su mente, entre otros. No obstante, la paz externa hace referencia a la paz del objeto, es decir; aquella que recae sobre un fenómeno cultural, jurídico, político y social.

\section{Conclusiones}

En la vida diaria de una sociedad existe una relación directa entre modelos y valores. Los modelos marcan y determinan las acciones concretas desarrolladas en la vida social, pero estas mismas acciones dejan de tener sentido en la mayoría de circunstancias si no están inspiradas en un valor reconocido. Esta conexión entre modelos y valores implica relaciones complejas, como lo es en gran medida la relación entre las personas. Hay que resaltar también que cuando un grupo de personas o una sociedad eligen un determinado modelo de actuación, implícitamente se adhiere a un determinado valor o valores.

Bajo este contexto, una clasificación de valores en la que cada valor se alinea a lo largo de un proceso continuo según su importancia se denomina "escala de valores", esto es la jerarquía de valores. En cuanto a los valores que se manifiestan en la convivencia ciudadana. Se considera que no existe una interacción positiva directa entre los miembros de las comunidades, la cual se limita a la práctica social formal, de manera que se asumen actitudes en ocasiones hostiles en cuanto promover la cooperación, el respeto y la justicia.

Atendiendo a las consideraciones anteriores, la gestión de la diversidad cultural para el fomento de valores en la convivencia ciudadana se encuentra en un nivel medianamente eficiente, dado que hay una disparidad entre los elementos o procesos que se toman en cuenta para promoverlos desde la práctica social, en este sentido, hay un vacío con respecto a los valores del respeto, la justicia y la honestidad; se ha percibido que no se establecen como parte de la práctica para orientar al individuo hacia su comportamiento social y familiar.

En consecuencia, existen algunos niveles de deficiencia en aspectos que se manifiestan desde la perspectiva del desarrollo formal de la conducta ciudadana. En este ámbito se pudo conocer que hay personas que no están comprometidas con los roles necesarios para formar ciudadanos a partir del respeto, la honestidad, la cooperación y la libertad de pensamientos, por tal motivo, se sustenta el desarrollo del objetivo de la investigación, el cual incluye una serie de actividades y procesos para el desarrollo del individuo y las comunidades, de manera que este a su vez pueda promover los valores desde su práctica social, familiar y cultural.

En tal sentido, deben realizarse convivencias en las que los productos esperados sean la reflexión y diálogo de cada uno de los estamentos sobre algunos temas; registros de percepciones y opiniones de los actores sobre los temas 
consultados previamente; revisión de los procesos sociales y de convivencia, la responsabilidad social y ambiental, y el respeto activo; toma de decisión de trabajar en el mejoramiento de la calidad de la convivencia ciudadana enfatizando la formación de valores y su importancia para la vida, entre otros aspectos. En efecto, se espera que las estrategias realizadas se integren al trabajo de las comunidades, tomando en cuenta la diversidad cultural y el proceso cotidiano de los miembros de las comunidades.

\section{Referencias bibliográficas}

Alonso, A. (2004). Mujeres en los medios, mujeres de los medios. Málaga, España: Icaria Editorial.

Carreras, Y. (2000). Cómo educar en valores. 7a Edición. Madrid, España: Narcea S.A.

Cortina, A. (2007), Ética aplicada y democracia radical. Navarra: Verbo Divino.

Duran S. (2010). Diversidad cultural y socialización organizacional del personal administrativo de las universidades privadas del municipio Maracaibo. [Tesis doctoral]. Maracaibo, Venezuela: Universidad Dr. Rafael Belloso Chacín.

(2015). Promoción de las instituciones educativas bajo el contexto de la interculturalidad. Revista Arbitrada Formación Gerencial, 13(2). Recuperado de: http://www.produccioncientifica. luz.edu.ve/index.php/rafg/article/ view/19990

Fuenmayor, A., Cárdenas, S., y Hernández, R. (2016). Emprendimiento como proceso de responsabilidad social en instituciones de educación superior en Colombia y Venezuela. Desarrollo gerencial, 8(2), 58-75. Recuperado de: htttp://revistas.unisimon.edu. co/index.php/desarrollogerencial/ article/view/2560
Parra, M. (2014). Diversidad Cultural para promover el desarrollo de habilidades sociales en educación superior. Cultura educación y sociedad, 5(1), 55-67. Recuperado de: http:// revistascientificas.cuc.edu.co/index. $\mathrm{php} / \mathrm{culturaeducacionysociedad/}$ article/view/995

Parra M. y Márceles, V. (2015). Potenciación de habilidades para el desarrollo de emprendedores exitosos en el contexto universitario. Universidad del Zulia. Departamento de Ciencias Humanas. Recuperado de: http://produccioncientificaluz. org/index.php/opcion/article/ view/20052/19978

Durán, S.E., García, J. y Prieto R. (2017). Influencia de la calidad de vida en el rendimiento del estudiante universitario. Calidad de vida, inclusión social y bienestar humano, 1(222). Recuperado de: https://www.researchgate. net/publication/317098368. Influencia_de_la_Calidad de_Vida_ en_el_rendimiento_del estudiante universitario.

Paz, M.A. (2016). Nuevas perspectivas ante los desafíos académicos. Vol. 1. Universidad del Zulia, Corporación Universitaria Latinoamericana y Universidad de la Guajira. Recuperado de: https://www. researchgate.net/profile/Sonia_ 
Duran/publication/317098580 DESARROLLO_ENDOGENO_ FACTOR_GENERADOR DE_CALIDAD_DE_VIDA/ links/5925d560458515e3d4527bf2/ DESARROLLO-ENDOGENO-FACTORGENERADOR-DE-CALIDAD-DEVIDA.pdf

Esté, A. (2002). Valores y referentes. Caracas, Venezuela: Universidad Central de Venezuela.

Fernández, J. (2001). El mundo de los valores. $2^{a}$ ed. Colombia: El Búho.

García Guiliany, J.; Duran, S.E. y Parra. M.A. (2017). Dirección estratégica del talento humano para el fomento de valores en los cuerpos policiales venezolanos. Espacios, 38(32), 1628. Recuperado de: http://www. revistaespacios.com/a17v38n32/ a17v38n32p16.pdf.

Hernández, O. y Prieto, R. (s.f.). Cultura organizacional en cooperativas del sector agrícola en el departamento del Atlántico. Diálogo de saberes, 6.

Kreitner, R., y Kinicki, Á. (2006). Comportamiento organizacional. México: Mc Graw Hill.

Martinneli, M., (2004). Conversando sobre educación en valores humanos. Brasil: Editorial Peiropolis.

Montesinos, M.P. (2016). Claves para pensar la diversidad cultural y la inclusión educativa. Educación, Lenguaje y Sociedad, 9(9).
Newstrom, J. (2011). Comportamiento humano en el trabajo. 13a ed. México: Mc Graw Hill.

Prieto, R.; Emonet, P.; García, J. y González, D. (2015). Cambio organizacional como estrategia de gestión en las empresas mixtas del sector petrolero. Revista de Ciencias Sociales. 21(3), 386-402.

Robbins, S. (2014). Fundamentos del comportamiento organizacional. México: Pearson.

Torralba, M. (2001). Diversidad y educación física. Revista Tándem. 5, 32-48.

Thomas, W. (2004). El valor de la libertad y los valores. Recuperado de: www. esposiblelaesperanza.com/index. php?...valores-thomas...

Zapata Domínguez, A. (2008). Gestión de la cultura organizacional. Bases conceptuales para su implementación (con Alfonso Rodríguez). Cali: Universidad del Valle.

Carr-Ruffino, N. (2013). Managing Diversity. 9a ed. San Francisco, Estados Unidos: San Francisco State University, Pearson Learning Solutions. 